\title{
A REMARK ON AN EQUATION OF WAVE MAPS TYPE WITH VARIABLE COEFFICIENTS
}

\author{
DAN-ANDREI GEBA
}

\begin{abstract}
We obtain a sharp local well-posedness result for an equation of wave maps type with variable coefficients. The proof relies on a recent construction ([2]) of curved versions for $X^{s, \theta}$ spaces.
\end{abstract}

\section{Introduction}

In this paper we study the low regularity well-posedness for a variable coefficient wave maps type equation. This can be viewed as a continuation of recent work ([2]) that investigated the same question in the general class of quadratic nonlinear wave equations on curved background.

A function $u: \mathbb{R}^{1+n} \rightarrow M$, from the Minkowski space-time into a Riemannian manifold $(M, h)$, is called a wave map if it is a critical point for the Lagrangian

$$
L(u)=\int_{\mathbb{R}^{1+n}}<\partial u, \partial u>d t d x
$$

where

$$
<\partial u, \partial u>=h_{J K} \partial_{\alpha} u^{J} \partial^{\alpha} u^{K}
$$

in local coordinates on $\mathrm{M}$, with $\partial^{t}=-\partial_{t}$ and $\partial^{x}=\partial_{x}{ }^{1}$. The associated EulerLagrange equation has the simplified form:

$$
\square u^{I}=\Gamma_{J K}^{I}(u) Q_{0}\left(u^{J}, u^{K}\right)
$$

where $\square=\partial_{\alpha} \partial^{\alpha}=-\partial_{t}^{2}+\Delta$ is the wave operator, $u=\left(u^{1}, u^{2}, \ldots, u^{N}\right), \Gamma_{J K}^{I}$ are the Christoffel symbols of $M$ in the local chart, $N=\operatorname{dim} M$ and $Q_{0}$ is the null form

$$
Q_{0}(v, w)=\partial_{\alpha} v \cdot \partial^{\alpha} w=-\partial_{t} v \cdot \partial_{t} w+\sum_{i=1}^{n} \partial_{i} v \cdot \partial_{i} w
$$

The initial value problem for (1) in $\mathbb{R}^{n+1}$, subject to the initial data

$$
u(0, x)=u_{0}(x), \quad \partial_{t} u(0, x)=u_{1}(x)
$$

is known to be locally well-posed for

$$
\left(u_{0}, u_{1}\right) \in H^{s}\left(\mathbb{R}^{n}\right) \times H^{s-1}\left(\mathbb{R}^{n}\right), \quad s>\frac{n}{2}
$$

This range is optimal and it was obtained in a series of papers by KlainermanMachedon [4] ( $n \geq 4)$, Klainerman-Selberg [8] $(n \geq 2)$, and Keel-Tao [3] $(n=1)$.

Received by the editors April 28, 2008

${ }^{1}$ Throughout this paper we assume the standard convention on summation. 
These results should be compared with the ones for general quadratic semilinear wave equations (Ponce-Sideris [10], Foschi -Klainerman [1], Tataru [14])

$$
\square u=q^{i j}(u) \partial_{i} u \partial_{j} u
$$

in which the Cauchy problem is known to be locally well-posed for

$$
s>\max \left\{\frac{n}{2}, \frac{n+5}{4}\right\}
$$

The gap in regularity between the two equations, noticeable only for dimensions $2 \leq n \leq 4$, has to do with the improved cancellation properties of $Q_{0}$. In loose terms, the null form damps the parallel interactions of the two functions, but preserves the transverse ones. One can see this by looking at its Fourier symbol

$$
\widetilde{Q}_{0}(\tau, \xi, \sigma, \eta)=\tau \sigma-\xi \cdot \eta=\left((\tau+\sigma)^{2}-|\xi+\eta|^{2}\right)-\left(\tau^{2}-|\xi|^{2}\right)-\left(\sigma^{2}-|\eta|^{2}\right)
$$

which vanishes if both $(\tau, \xi)$ and $(\sigma, \eta)$ are on the cone and collinear.

For a better motivation of our problem, let us consider also the associated quasilinear wave equation

$$
\square_{g(u)} u=q^{i j}(u) \partial_{i} u \partial_{j} u
$$

with $\square_{g}=g^{i j} \partial_{i} \partial_{j}$ (the summation occurs from 0 to $n$ and the index 0 stands for the time variable). To insure hyperbolicity, we assume that the matrix $g^{i j}$ has signature $(1, n)$ and the time level sets $\left\{x_{0}=\right.$ const $\}$ are space-like (i.e., $\left.g^{00}>0\right)$. For simplicity, we take $g^{00} \equiv 1$.

Sharp local well-posedness holds for (7) only in the $2+1$ and $3+1$ dimensional cases (Smith-Tataru [12], Lindblad [9]; see also the results of Klainerman-Rodnianski [5], [6], [7], for Einstein vacuum equations). For higher dimensions, it is not known yet whether the range (5) applies to (7) too, or if one needs to impose additional conditions on $s$.

A first step in answering this question was taken in [2], where the related semilinear problem on a curved background

$$
\square_{g} u=q^{i j}(u) \partial_{i} u \partial_{j} u
$$

was investigated. It was shown that for metrics satisfying $\partial^{2} g \in L_{t}^{2} L_{x}^{\infty}{ }^{2}$, the Cauchy problem for (8) is locally well-posed in

$$
H^{s}\left(\mathbb{R}^{4}\right) \times H^{s-1}\left(\mathbb{R}^{4}\right), \quad s>\frac{9}{4}
$$

matching the above range, (5).

In this article we consider the variable coefficient version of (1), namely

$$
\square_{g} u^{I}=\Gamma_{J K}^{I}(u) Q_{0}^{g}\left(u^{J}, u^{K}\right)
$$

where the corresponding null form $Q_{0}^{g}$ is defined as

$$
Q_{0}^{g}(u, v)=g^{\alpha \beta} \partial_{\alpha} u \partial_{\beta} v=\frac{1}{2}\left(\square_{g}(u v)-u \square_{g} v-v \square_{g} u\right)
$$

We are able to prove that in certain dimensions, under reasonable regularity conditions for the metric $g$, the local well-posedness theories for (1) and (9) coincide:

\footnotetext{
${ }^{2}$ We make the convention in the sequel that $L^{p} L^{q}=L_{t}^{p} L_{x}^{q}$, while $L^{p}=L_{t, x}^{p}$, with the exception of fixed time estimates.
} 
Theorem 1.1. Let $3 \leq n \leq 5$ and assume $\partial^{2} g \in L^{2} L^{\infty}$. The Cauchy problem associated to (9) is locally well-posed in $H^{s}\left(\mathbb{R}^{n}\right) \times H^{s-1}\left(\mathbb{R}^{n}\right)$ for $s>\frac{n}{2}$.

Remark 1.2. The hypothesis on the regularity of the metric $g$ is related to the fact that, for both (8) and (9), we have to control $X^{s, \theta}$ norms, which are in fact $L^{2} L^{2}$ norms. A typical cross term to estimate is

$$
\left\|\partial^{2} g \cdot u\right\|_{L^{2} L^{2}} \lesssim\left\|\partial^{2} g\right\|_{L^{2} L^{\infty}}\|u\|_{L^{\infty} L^{2}}
$$

One needs to compare this with the optimal regularity, $\partial^{2} g \in L^{1} L^{\infty}$ (Tataru [15]), required for the wave operator $\square_{g}$ to have the full range of Strichartz exponents. It is interesting to note that these regularities infer that $g$ is global Lipschitz.

Remark 1.3. The more challenging case of dimension $n=2$ is current work in progress, and will be addressed in a subsequent paper. An argument based only on techniques contained in this article yields local well-posedness of the initial value problem in $H^{s}\left(\mathbb{R}^{2}\right)$ for $s>\frac{11}{8}$.

The proof of Theorem 1.1 is based, as it is usually the case for these problems, on a fixed point argument that exhibits a considerable amount of similarities with the one in [2]. To be precise, all that is needed new for (9), in comparison to (8), is one new estimate and several extensions of previous ones to different settings.

Due to (10), and modulo the standard reduction to the case of small initial data and time interval set to $[-1,1]$, our result can be inferred from the following set of inequalities:

$$
\begin{aligned}
\left\|S\left(u_{0}, u_{1}\right)\right\|_{X} \lesssim\left\|\left(u_{0}, u_{1}\right)\right\|_{H^{s} \times H^{s-1}} \\
\left\|\square_{g}^{-1} H\right\|_{X} \lesssim\|H\|_{Y} \\
\left\|\square_{g} u\right\|_{Y} \lesssim\|u\|_{X} \\
\|u \cdot v\|_{X} \lesssim\|u\|_{X}\|v\|_{X} \\
\|\Gamma(u)\|_{X} \lesssim C\left(\|u\|_{L^{\infty}}\right)\left(1+\|u\|_{X}^{5}\right) \\
\|u \cdot w\|_{Y} \lesssim\|u\|_{X}\|w\|_{Y}
\end{aligned}
$$

where $C=C\left(\|u\|_{L^{\infty}}\right)$ is a constant that depends solely on the dimension $n$ and $\|u\|_{L^{\infty}}, X$ and $Y$ are suitably chosen Banach spaces, while $S\left(u_{0}, u_{1}\right)$ and $\square_{g}^{-1}$ are respectively the homogeneous and inhomogeneous solution operators

$$
\begin{aligned}
& \square_{g} S\left(u_{0}, u_{1}\right)=0, \quad S\left(u_{0}, u_{1}\right)(0)=u_{0}, \quad \partial_{t} S\left(u_{0}, u_{1}\right)(0)=u_{1} \\
& \square_{g}\left(\square_{g}^{-1} H\right)=H, \quad\left(\square_{g}^{-1} H\right)(0)=0, \quad \partial_{t}\left(\square_{g}^{-1} H\right)(0)=0
\end{aligned}
$$

Remark 1.4. 1. The new estimate which is needed in our analysis, but was absent in the one for equation (8), is (13).

2. Based on (10), (13), (14), and (16), one can infer immediately that

$$
\left\|Q_{0}^{g}(u, v)\right\|_{Y} \lesssim\|u\|_{X}\|v\|_{X}
$$

This is the variable coefficient version of a certain null-form estimate of KlainermanMachedon, which has been previously obtained by Sogge [13], Sogge-Smith [11], and Tataru [16], under various regularity assumptions for the metric $g$. 
Remark 1.5. 1. (11)-(12) have already been proved in [2], in the context of our problem (i.e., for $3 \leq n \leq 5$ ).

2. [2] contains the proof of (14)-(16) for the $4+1$ dimensional case; here we extend them to $3+1$, respectively $5+1$ dimensions.

In the next section, for completeness, we will reintroduce the notations, definitions, and results from [2] that will be needed in our analysis, while in the last section, we will discuss the estimates mentioned in the above remarks.

\section{Curved $X^{s, \theta}$ spaces: definitions, notations and properties}

We introduce first the Banach spaces that will be used in our argument:

$$
X=X^{s, \theta} \quad Y=X^{s-1, \theta-1}
$$

where

Definition 2.1. (Definition 2.1 in [2])

For $0<\theta<1$ and $s \in \mathbb{R}, X^{s, \theta}$ is the space of functions $u \in L^{2}\left(-1,1 ; H^{s}\left(\mathbb{R}^{n}\right)\right)$ for which

$$
\|u\|_{X^{s, \theta}}^{2}=\inf \left\{\sum_{\lambda=1}^{\infty} \sum_{d=1}^{\lambda}\left\|u_{\lambda, d}\right\|_{X_{\lambda, d}^{s, \theta}}^{2} ; u=\sum_{\lambda=1}^{\infty} \sum_{d=1}^{\lambda} S_{\lambda} u_{\lambda, d}\right\}<\infty
$$

with $\lambda, d$ taking dyadic values and

$$
\left\|u_{\lambda, d}\right\|_{X_{\lambda, d}^{s, \theta}}=\lambda^{s} d^{\theta}\left\|u_{\lambda, d}\right\|_{L^{2}}+\lambda^{s-1} d^{\theta-1}\left\|\square_{g_{<\sqrt{\lambda}}} u_{\lambda, d}\right\|_{L^{2}}
$$

We define negative index spaces by setting, again for $0<\theta<1$ and $s \in \mathbb{R}$, the $X^{s-1, \theta-1}$ norm to equal

$$
\begin{aligned}
\|f\|_{X^{s-1, \theta-1}}^{2}=\inf \left\{\left\|f_{0}\right\|_{L^{2} H^{s-1}}^{2}+\sum_{\lambda=1}^{\infty} \sum_{d=1}^{\lambda}\left\|f_{\lambda, d}\right\|_{X_{\lambda, d}^{s, \theta}}^{2} ;\right. \\
\left.\quad f=f_{0}+\sum_{\lambda=1}^{\infty} \sum_{d=1}^{\lambda} \square_{g_{<\sqrt{\lambda}}} S_{\lambda} f_{\lambda, d}\right\}<\infty
\end{aligned}
$$

Remark 2.2. $S_{\lambda}, S_{<\lambda}$ and $S_{\geq \lambda}$ are the usual multipliers associated with a LittlewoodPaley decomposition. All but one frequency localizations are with respect to the spatial variables (i.e., $S_{\lambda}=S_{\lambda}\left(D_{x}\right)$ ). The exception is the localization of the metric $g$, which is truncated using space-time multipliers. For this to work, we extend $g$ to have similar properties in all of $\mathbb{R}^{n+1}$. In this case, $S_{\lambda}=S_{\lambda}\left(D_{t, x}\right)$.

We start by recording

Lemma 2.3. (Lemma 2.4 in [2])

The following estimate holds:

$$
\lambda^{s-1} d^{\theta}\left\|\nabla S_{\lambda} u\right\|_{L^{2}}+\lambda^{s-1} d^{\theta-1}\left\|\square_{g_{<\sqrt{\lambda}}} S_{\lambda} u\right\|_{L^{2}} \lesssim\|u\|_{X_{\lambda, d}^{s, \theta}}
$$


This result allows us to replace the $X_{\lambda, d}^{s, \theta}$ norm in the Definition 2.1 by

$$
\|u\|_{\tilde{X}_{\lambda, d}^{s, \theta}}=\lambda^{s-1} d^{\theta}\|\nabla u\|_{L^{2}}+\lambda^{s-1} d^{\theta-1}\left\|\square_{g_{<\sqrt{\lambda}}} u\right\|_{L^{2}}
$$

and to assume, without any restriction in generality, that the functions $u_{\lambda, d}$ and $f_{\lambda, d}$, appearing in the above definition, are localized at frequency $\lambda$. The presence of the gradient in this new, equivalent norm, is easier to handle in the context of the wave equation. An immediate consequence of this is the following

Lemma 2.4. (Corollary 2.8 in [2])

For $\theta>0$ the following energy inequality takes place

$$
\lambda^{s-1} d^{\theta-\frac{1}{2}}\left\|\nabla S_{\lambda} u\right\|_{L^{\infty} L^{2}} \lesssim\left\|S_{\lambda} u\right\|_{\tilde{X}_{\lambda, d}^{s, \theta}}
$$

As a result, for $\theta>\frac{1}{2}$ :

$$
\|u\|_{L^{\infty} H^{s}}+\left\|u_{t}\right\|_{L^{\infty} H^{s-1}} \lesssim\|u\|_{X^{s, \theta}}
$$

The next important issue to address is the truncation in frequency of the metric g. This is achieved by

Lemma 2.5. (Lemma 2.9 in [2])

If $\tilde{S}_{\lambda}$ is a spatial multiplier with slightly larger support (i.e., $S_{\lambda} \tilde{S}_{\lambda}=S_{\lambda}$ ), $0 \leq s \leq 3$, and the metric $g$ has regularity $\partial^{2} g \in L^{2} L^{\infty}$, the following fixed time estimate is true:

$$
\sum_{\lambda=1}^{\infty} \lambda^{2(s-1)}\left\|\tilde{S}_{\lambda}\left(g_{>\sqrt{\lambda}} u\right)(t)\right\|_{L^{2}}^{2} \lesssim\left(M\left(\left\|\partial^{2} g\right\|_{L_{x}^{\infty}}\right)(t)\right)^{2}\|u(t)\|_{H^{s-2}}^{2}
$$

with $M$ being the maximal function with respect to time. The dual estimate also holds:

$$
\left\|\sum_{\lambda=1}^{\infty} g_{>\sqrt{\lambda}}(t) \tilde{S}_{\lambda} f_{\lambda}(t)\right\|_{H^{2-s}}^{2} \lesssim \sum_{\lambda=1}^{\infty} \lambda^{2(1-s)}\left\|f_{\lambda}(t)\right\|_{L^{2}}^{2}
$$

As it turns out, the results listed so far are the only tools needed to prove the estimates for the homogeneous and inhomogeneous solution operators (i.e., (11) and (12)). We are left to investigate (13)-(16).

To go further, one needs to identify the link between the $X^{s, \theta}$ spaces of positive and negative indices:

Lemma 2.6. (Lemma 2.13 in [2])

For $0<\theta<\frac{1}{2}$ we have the following duality relation

$$
X^{-s,-\theta}=\left(X^{s, \theta}+L^{2} H^{s+\theta}\right)^{\prime}
$$

The next natural step is to relate the $X^{s, \theta}$ spaces to spaces relevant to Strichartz estimates. We have the following:

Lemma 2.7. (Corollary 3.2 in [2])

a) For $\theta>0$ and $(\sigma, p, q)$ verifying

$$
\sigma=-\frac{n}{2}+\frac{1}{p}+\frac{n}{q}, \quad \frac{2}{p}+\frac{n-1}{q} \leq \frac{n-1}{2}, \quad 2 \leq p \leq \infty, 2 \leq q<\infty
$$

we have

$$
\left\|S_{\lambda} \nabla u\right\|_{L^{p} L^{q}} \lesssim \lambda^{1-s-\sigma} d^{\frac{1}{2}-\theta}\|u\|_{X_{\lambda, d}^{s, \theta}}
$$


If additionally $\theta>\frac{1}{2}$ then

$$
\left\|S_{\lambda} \nabla u\right\|_{L^{p} L^{q}} \lesssim \lambda^{1-s-\sigma}\|u\|_{X^{s, \theta}}
$$

b) If instead

$$
\frac{2}{p}+\frac{n-1}{q} \geq \frac{n-1}{2}
$$

then

$$
\left\|S_{\lambda} \nabla u\right\|_{L^{p} L^{q}} \lesssim \lambda^{1-s-\sigma+\frac{1}{2}\left(\frac{2}{p}+\frac{n-1}{q}-\frac{n-1}{2}\right)} d^{\frac{1}{2}-\theta-\frac{1}{2}\left(\frac{2}{p}+\frac{n-1}{q}-\frac{n-1}{2}\right)}\|u\|_{X_{\lambda, d}^{s, \theta}}
$$

Remark 2.8. In the above embeddings, one can use also the index $q=\infty$. We will rely in particular on the triplets

$$
\left(-\frac{n}{2}, \infty, \infty\right) \quad\left(-\frac{n-1}{2}, 2, \infty\right)
$$

noting that for $n=3$ and $(\sigma, p, q)=(-1,2, \infty)$, one loses in the previous bounds either $a \ln \lambda$ or $\lambda^{\epsilon}$, with $\epsilon>0$ arbitrary small. However, this loss is harmless because it is covered by the strict inequalities imposed on the exponents. Therefore we can ignore it.

For a finer analysis, that is needed in the last section, we introduce also the norm (see section 3 in [2])

$$
\|u\|_{\tilde{X}_{\lambda,<d}^{s, \theta}}^{2}=\inf \left\{\sum_{h=1}^{d}\left\|u_{h}\right\|_{\tilde{X}_{\lambda, h}^{s, \theta}}^{2} ; u=\sum_{h=1}^{d} u_{h}\right\} \leq\|u\|_{\tilde{X}_{\lambda, d}^{s, \theta}}^{2}
$$

For $d=\lambda$ we use instead the notation $\tilde{X}_{\lambda}^{s, \theta}$. A straightforward calculation yields

$$
\|u\|_{X^{s, \theta}}^{2}=\inf \left\{\sum_{\lambda=1}^{\infty}\left\|S_{\lambda} u_{\lambda}\right\|_{\tilde{X}_{\lambda}^{s, \theta}}^{2} ; u=\sum_{\lambda=1}^{\infty} S_{\lambda} u_{\lambda}\right\}
$$

In this new context, for $\theta>0$ and $(\sigma, p, q)$ as in part a) of Lemma 2.7, we also have (Corollary 3.4 in [2]):

$$
\left\|S_{\lambda} \nabla u\right\|_{L^{p} L^{q}} \lesssim \lambda^{1-s-\sigma} \max \left\{1, d^{\frac{1}{2}-\theta}\right\}\|u\|_{X_{\lambda,<d}^{s, \theta}}
$$

Finally, we make the following

Remark 2.9. All the results mentioned in this section are proved in [2], independent of dimension $n$.

\section{Conclusion: Proof of the estimates (13)-(16)}

We start by proving (13), which, as mentioned in the first section, is one of the novelties of this paper, being specifically needed for our problem, (9):

Proposition 3.1. For $\theta>\frac{1}{2}$ and $0 \leq s \leq 3$, the following mapping holds:

$$
\square_{g}: X^{s, \theta} \rightarrow X^{s-1, \theta-1}
$$


Proof. We use the decomposition

$$
u=\sum_{\lambda=1}^{\infty} \sum_{d=1}^{\lambda} S_{\lambda} u_{\lambda, d}=\sum_{\lambda=1}^{\infty} S_{\lambda} u_{\lambda}
$$

with

$$
u_{\lambda}=\sum_{d=1}^{\lambda} u_{\lambda, d}
$$

Then one can write

$$
\square_{g} u=\sum_{\lambda=1}^{\infty} \square_{g_{>\sqrt{\lambda}}} S_{\lambda} u_{\lambda}+\sum_{\lambda=1}^{\infty} \sum_{d=1}^{\lambda} \square_{g_{<\sqrt{\lambda}}} S_{\lambda} u_{\lambda, d}
$$

Using Definition 2.1, Lemma 2.5, and (26), we can infer

$$
\begin{aligned}
\left\|\square_{g} u\right\|_{X^{s-1, \theta-1}}^{2} & \lesssim\left\|\sum_{\lambda=1}^{\infty} \square_{g_{>\sqrt{\lambda}}} S_{\lambda} u_{\lambda}\right\|_{L^{2} H^{s-1}}^{2}+\sum_{\lambda=1}^{\infty} \sum_{d=1}^{\lambda}\left\|u_{\lambda, d}\right\|_{X_{\lambda, d}^{s, \theta}}^{2} \\
& \lesssim \sum_{\lambda=1}^{\infty}\left\|\nabla S_{\lambda} u_{\lambda}\right\|_{L^{\infty} H^{s-1}}^{2}+\|u\|_{X^{s, \theta}}^{2} \lesssim\|u\|_{X^{s, \theta}}^{2}
\end{aligned}
$$

For (14)-(16), at this moment, our arguments for $3+1$, respectively $5+1$ dimensions do not stray far from the $4+1$ dimensional proof, given in [2]. This is why we discuss here only the relevant differences and the corresponding numerology.

A key ingredient of this analysis is to have good $X^{s, \theta}$ control on bilinear structures that involve functions localized at different frequencies. The $4+1$ dimensional case is handled by Lemmas 3.5 and 3.6 in [2]. Another contribution of our paper is that it optimizes that argument (i.e., we obtain improved bounds for some estimates), allowing for these results to be written in a more compact form.

Lemma 3.2. Let $\theta>0$ and $n \geq 3$.

i) The following estimates hold

$$
\begin{aligned}
&\left\|\partial_{t t} S_{\lambda} u\right\|_{L^{2} L^{\infty}} \lesssim \lambda^{\frac{n+3}{2}-s} d^{\frac{1}{2}-\theta}\|u\|_{\tilde{X}_{\lambda, d}^{s, \theta}} \\
&\left\|\partial_{t t} S_{\lambda} u\right\|_{L^{2} L^{\infty}} \lesssim \lambda^{\frac{n+3}{2}-s} \max \left\{1, d^{\frac{1}{2}-\theta}\right\}\|u\|_{\tilde{X}_{\lambda,<d}^{s, \theta}}
\end{aligned}
$$

ii) For $\mu \leq d \leq \lambda$, we have

$$
\begin{aligned}
\left\|S_{\mu} v S_{\lambda} u\right\|_{\tilde{X}_{\lambda, d}^{s, \theta}} & \lesssim\left(\left\|S_{\mu} v\right\|_{L^{\infty}}+\min \left\{d^{-1}\left\|\nabla S_{\mu} v\right\|_{\left.L^{\infty}, d^{-\frac{1}{2}}\left\|\nabla S_{\mu} v\right\|_{L^{2} L^{\infty}}\right\}+}\right.\right. \\
& \left.+\lambda^{-1} d^{-\frac{1}{2}}\left\|\partial_{t t} S_{\mu} v\right\|_{L^{2} L^{\infty}}\right)\left\|S_{\lambda} u\right\|_{\tilde{X}_{\lambda, d}^{s, \theta}} \\
& \lesssim \mu^{\frac{n}{2}-s} \max \left\{1, \mu^{\frac{1}{2}-\theta}\right\}\left\|S_{\mu} v\right\|_{\tilde{X}_{\mu}^{s, \theta}}^{s, S_{\lambda} u \|_{\tilde{X}_{\lambda, d}^{s, \theta}}}
\end{aligned}
$$


iii) For $\mu \leq \lambda$, we have

$$
\begin{aligned}
\left\|S_{\mu} v S_{\lambda} u\right\|_{\tilde{X}_{\lambda, \mu}^{s, \theta}} & \lesssim\left(\left\|S_{\mu} v\right\|_{L^{\infty}}+\max \left\{\mu^{\theta-1}, \mu^{-\frac{1}{2}}\right\}\left\|\nabla S_{\mu} v\right\|_{L^{2} L^{\infty}}+\right. \\
& \left.+\max \left\{\mu^{\theta-2}, \mu^{-\frac{3}{2}}\right\}\left\|\partial_{t t} S_{\mu} v\right\|_{L^{2} L^{\infty}}\right)\left\|S_{\lambda} u\right\|_{\tilde{X}_{\lambda,<\mu}^{s, \theta}} \\
& \lesssim \mu^{\frac{n-1}{2}+\theta-s} \max \left\{1, \mu^{1-2 \theta}\right\}\left\|S_{\mu} v\right\|_{\tilde{X}_{\mu}^{s, \theta}}\left\|S_{\lambda} u\right\|_{\tilde{X}_{\lambda,<\mu}^{s, \theta}}
\end{aligned}
$$

Proof. The main tools used in this proof are the energy estimate (25) and the embeddings contained in Lemma 2.7 .

For (38) and (39), we combine (30) and (35) with the observation, based on $g^{00} \equiv 1$, that

$$
\left(\partial_{t t}-\square_{g_{<\sqrt{\lambda}}}\right) S_{\lambda} \approx \lambda \nabla S_{\lambda}
$$

(40) and (41) have similar arguments. The strategy is to estimate the $L^{2}$ norm of the cross terms arising from Leibnitz's rule either by a $L^{\infty} L^{2} \cdot L^{2} L^{\infty}$ product or a $L^{2} L^{2} \cdot L^{\infty} L^{\infty}$ one. We focus on (41). The first two cross terms can be estimated directly, using (35):

$$
\begin{aligned}
& \lambda^{s-1} \mu^{\theta}\left\|\nabla S_{\mu} v \cdot S_{\lambda} u\right\|_{L^{2}} \lesssim \frac{\max \left\{\mu^{\theta}, \mu^{\frac{1}{2}}\right\}}{\lambda}\left\|\nabla S_{\mu} v\right\|_{L^{2} L^{\infty}}\left\|S_{\lambda} u\right\|_{\tilde{X}_{\lambda,<\mu}^{s, \theta}} \\
& \lambda^{s-1} \mu^{\theta}\left\|S_{\mu} v \cdot \nabla S_{\lambda} u\right\|_{L^{2}} \lesssim \max \left\{\mu^{\theta}, \mu^{\frac{1}{2}}\right\}\left\|S_{\mu} v\right\|_{L^{2} L^{\infty}}\left\|S_{\lambda} u\right\|_{\tilde{X}_{\lambda,<\mu}^{s, \theta}}
\end{aligned}
$$

More delicate terms appear when $\square_{g_{<\sqrt{\lambda}}}$ acts on the product $S_{\mu} v S_{\lambda} u$. The first two are easier to estimate than the one involving $\square_{g_{<\sqrt{\lambda}}} S_{\mu} v$ :

$$
\begin{aligned}
& \lambda^{s-1} \mu^{\theta-1}\left\|S_{\mu} v \cdot \square_{g_{<\sqrt{\lambda}}} S_{\lambda} u\right\|_{L^{2}} \lesssim\left\|S_{\mu} v\right\|_{L^{\infty}}\left\|S_{\lambda} u\right\|_{\tilde{X}_{\lambda,<\mu}^{s, \theta}} \\
& \lambda^{s-1} \mu^{\theta-1}\left\|g_{<\sqrt{\lambda}} \nabla S_{\mu} v \cdot \nabla S_{\lambda} u\right\|_{L^{2}} \lesssim \max \left\{\mu^{\theta-1}, \mu^{-\frac{1}{2}}\right\}\left\|\nabla S_{\mu} v\right\|_{L^{2} L^{\infty}}\left\|S_{\lambda} u\right\|_{\tilde{X}_{\lambda,<\mu}^{s, \theta}}
\end{aligned}
$$

For the last term, let us consider the decomposition

$$
u=\sum_{h=1}^{\mu} u_{h}
$$

as in the definition of the $\tilde{X}_{\lambda,<\mu}^{s, \theta}$ spaces. We can infer as follows

$$
\begin{aligned}
& \lambda^{s-1} \mu^{\theta-1}\left\|\square_{g_{<\sqrt{\lambda}}}\left(S_{\mu} v\right) \cdot S_{\lambda} u\right\|_{L^{2}} \lesssim \\
& \lesssim \lambda^{s-1} \mu^{\theta-1}\left(\left\|\partial_{t t} S_{\mu} v\right\|_{L^{2} L^{\infty}}+\mu\left\|\nabla S_{\mu} v\right\|_{L^{2} L^{\infty}}\right)\left\|S_{\lambda} u\right\|_{L^{\infty} L^{2}} \\
& \lesssim \frac{\mu^{\theta-1}}{\lambda}\left(\left\|\partial_{t t} S_{\mu} v\right\|_{L^{2} L^{\infty}}+\mu\left\|\nabla S_{\mu} v\right\|_{L^{2} L^{\infty}}\right)\left(\sum_{h=1}^{\mu} \frac{1}{h^{2 \theta-1}} \cdot \sum_{h=1}^{\mu} \lambda^{2 s} h^{2 \theta-1}\left\|S_{\lambda} u_{h}\right\|_{L^{\infty} L^{2}}^{2}\right)^{\frac{1}{2}} \\
& \lesssim \frac{\mu^{\theta-1}}{\lambda} \max \left\{1, \mu^{\frac{1}{2}-\theta}\right\}\left(\left\|\partial_{t t} S_{\mu} v\right\|_{L^{2} L^{\infty}}+\mu\left\|\nabla S_{\mu} v\right\|_{L^{2} L^{\infty}}\right)\left(\sum_{h=1}^{\mu}\left\|S_{\lambda} u_{h}\right\|_{\tilde{X}_{\lambda, h}^{s, \theta}}^{2}\right)^{\frac{1}{2}}
\end{aligned}
$$

which is obviously the right bound.

We are now ready to prove the remaining main estimates: 
Proposition 3.3. Let $n \geq 3$ and $\frac{1}{2}<\theta<s-\frac{n-1}{2}$. Then:

$$
\begin{aligned}
& \|u \cdot v\|_{X^{s, \theta}} \lesssim\|u\|_{X^{s, \theta}}\|v\|_{X^{s, \theta}} \\
& \|u \cdot w\|_{X^{s-1, \theta-1}} \lesssim\|u\|_{X^{s, \theta}}\|w\|_{X^{s-1, \theta-1}} \\
& \|\Gamma(u)\|_{X^{s, \theta}} \lesssim C\left(\|u\|_{L^{\infty}}\right)\left(1+\|u\|_{X^{s, \theta}}^{5}\right)
\end{aligned}
$$

Proof. We use decompositions identical with the ones in (37):

$$
u=\sum_{\lambda=1}^{\infty} \sum_{d=1}^{\lambda} S_{\lambda} u_{\lambda, d}=\sum_{\lambda=1}^{\infty} S_{\lambda} u_{\lambda}, \quad v=\sum_{\lambda=1}^{\infty} \sum_{d=1}^{\lambda} S_{\lambda} v_{\lambda, d}=\sum_{\lambda=1}^{\infty} S_{\lambda} v_{\lambda}
$$

It follows that

$$
u v=\sum_{\mu=1}^{\infty} \sum_{\lambda_{1}=1}^{\infty} \sum_{\lambda_{2}=1}^{\infty} S_{\mu}\left(S_{\lambda_{1}} u_{\lambda_{1}} S_{\lambda_{2}} v_{\lambda_{2}}\right)
$$

The nontrivial interactions in the above sum are only when $\max \left\{\lambda_{1}, \lambda_{2}\right\} \approx \mu$ or $\lambda_{1} \approx \lambda_{2} \gtrsim \mu$. These are handled by using (40) and (41) to claim

$$
\begin{gathered}
\left\|S_{\lambda} u_{\lambda} S_{\lambda} v_{\lambda}\right\|_{X_{\mu, \mu}^{s, \theta}} \lesssim \mu^{s+\theta-2} \lambda^{\frac{n+3}{2}-2 s}\left\|u_{\lambda}\right\|_{\tilde{X}_{\lambda}^{s, \theta}}\left\|v_{\lambda}\right\|_{\tilde{X}_{\lambda}^{s, \theta}} \\
\left\|S_{\lambda} u_{\lambda} S_{\mu} v_{\mu}\right\|_{\tilde{X}_{\lambda}^{s, \theta}}^{2} \lesssim \mu^{n-1+2 \theta-2 s}\left\|v_{\mu}\right\|_{\tilde{X}_{\mu}^{s, \theta}}^{2} \sum_{d=1}^{\lambda}\left\|u_{\lambda, d}\right\|_{\tilde{X}_{\lambda, d}^{s, \theta}}^{2}
\end{gathered}
$$

It turns out that this is all that is needed to infer (42) (see also Proposition 3.7 in [2]).

Using the duality relation (29) and the fact that $s>\frac{n}{2}$, one can reduce (43) to

$$
X^{s, \theta} \cdot X^{1-s, 1-\theta} \subset X^{1-s, 1-\theta}+L^{2} H^{2-s-\theta}
$$

which is then treated by considering decompositions as above. In this case, there are three possible interactions, one of which requires a different analysis than the one of Proposition 3.8 in [2]. This is the term $S_{\mu}\left(S_{\lambda} u_{\lambda} S_{\lambda} v_{\lambda}\right)$ which needs to be estimated in the space $L^{2} H^{2-s-\theta}$.

For $n \geq 4$, (35) allows us to show that

$$
\begin{aligned}
\left\|S_{\lambda} u_{\lambda} S_{\lambda} v_{\lambda}\right\|_{L^{2} L^{\frac{n-1}{n-2}}} & \lesssim\left\|S_{\lambda} u_{\lambda}\right\|_{L^{4} L^{\frac{2(n-1)}{n-2}}}\left\|S_{\lambda} v_{\lambda}\right\|_{L^{4} L^{\frac{2(n-1)}{n-2}}} \\
& \lesssim \lambda^{\theta-1+\frac{1}{n-1}}\left\|u_{\lambda}\right\|_{\tilde{X}_{\lambda}^{s, \theta}}\left\|v_{\lambda}\right\|_{\tilde{X}_{\lambda}^{1-s, 1-\theta}}
\end{aligned}
$$

Sobolev embeddings can then be used to obtain

$$
\left\|S_{\mu}\left(S_{\lambda} u_{\lambda} S_{\lambda} v_{\lambda}\right)\right\|_{L^{2} H^{2-s-\theta}} \lesssim \mu^{2-s-\theta+\frac{n(n-3)}{2(n-1)}} \lambda^{\theta-1+\frac{1}{n-1}}\left\|u_{\lambda}\right\|_{\tilde{X}_{\lambda}^{s, \theta}}\left\|v_{\lambda}\right\|_{\tilde{X}_{\lambda}^{1-s, 1-\theta}}
$$

which agrees with the numerology included in the hypothesis.

The case $n=3$ is a little more delicate and we need (32) to infer

$$
\begin{aligned}
\left\|S_{\lambda} u_{\lambda} S_{\lambda} v_{\lambda}\right\|_{L^{2} L^{\frac{2}{1+2 \epsilon}}} & \lesssim\left\|S_{\lambda} u_{\lambda}\right\|_{L^{4} L^{\frac{4}{1-4 \epsilon}}}\left\|S_{\lambda} v_{\lambda}\right\|_{L^{4} L^{\frac{4}{1+8 \epsilon}}} \\
& \lesssim \lambda^{-\epsilon}\left\|u_{\lambda}\right\|_{\tilde{X}_{\lambda}^{s, \theta}}\left\|v_{\lambda}\right\|_{\tilde{X}_{\lambda}^{1-s, 1-\theta}}
\end{aligned}
$$

Again by Sobolev embeddings we can conclude that 


$$
\left\|S_{\mu}\left(S_{\lambda} u_{\lambda} S_{\lambda} v_{\lambda}\right)\right\|_{L^{2} H^{2-s-\theta}} \lesssim \mu^{2-s-\theta+3 \epsilon} \lambda^{-\epsilon}\left\|u_{\lambda}\right\|_{\tilde{X}_{\lambda}^{s, \theta}}\left\|v_{\lambda}\right\|_{\tilde{X}_{\lambda}^{1-s, 1-\theta}}
$$

The proof of (44) follows the same lines as the one in Proposition 3.9 from [2].

\section{Acknowledgements}

I am grateful to Daniel Tataru for suggesting this problem and for many stimulating

discussions. Also, I would like to thank the referee for many helpful suggestions.

\section{References}

[1] D. Foschi and S. Klainerman, Bilinear space-time estimates for homogeneous wave equations, Ann. Sci. École Norm. Sup. (4) 33 (2000), no. 2, 211-274.

[2] D.-A. Geba and D. Tataru, Gradient NLW on curved background in $4+1$ dimensions, Internat. Math. Res. Notices 2008 (2008) rnn108-58.

[3] M. Keel and T. Tao, Local and global well-posedness of wave maps on $\mathbf{R}^{1+1}$ for rough data, Internat. Math. Res. Notices (1998), no. 21, 1117-1156.

[4] S. Klainerman and M. Machedon, Smoothing estimates for null forms and applications, Duke Math. J. 81 (1995), no. 1, 99-133 (1996). A celebration of John F. Nash, Jr.

[5] S. Klainerman and I. Rodnianski, Rough solutions of the Einstein vacuum equations, C. R. Math. Acad. Sci. Paris 334 (2002), no. 2, 125-130.

[6] —, Ricci defects of microlocalized Einstein metrics, J. Hyperbolic Differ. Equ. 1 (2004), no. $1,85-113$.

[7] — The causal structure of microlocalized rough Einstein metrics, Ann. of Math. (2) 161 (2005), no. 3, 1195-1243.

[8] S. Klainerman and S. Selberg, Remark on the optimal regularity for equations of wave maps type, Comm. Partial Differential Equations 22 (1997), no. 5-6, 901-918.

[9] H. Lindblad, Counterexamples to local existence for quasilinear wave equations, Math. Res. Lett. 5 (1998), no. 5, 605-622.

[10] G. Ponce and T. C. Sideris, Local regularity of nonlinear wave equations in three space dimensions, Comm. Partial Differential Equations 18 (1993), no. 1-2, 169-177.

[11] H. F. Smith and C. D. Sogge, Null form estimates for $(1 / 2,1 / 2)$ symbols and local existence for a quasilinear Dirichlet-wave equation, Ann. Sci. École Norm. Sup. (4) 33 (2000), no. 4, 485-506.

[12] H. F. Smith and D. Tataru, Sharp local well-posedness results for the nonlinear wave equation, Ann. of Math. (2) 162 (2005), no. 1, 291-366.

[13] C. D. Sogge, On local existence for nonlinear wave equations satisfying variable coefficient null conditions, Comm. Partial Differential Equations 18 (1993), no. 11, 1795-1821.

[14] D. Tataru, On the equation $\square u=|\nabla u|^{2}$ in $5+1$ dimensions, Math. Res. Lett. 6 (1999), no. 5-6, 469-485.

[15] - Strichartz estimates for second order hyperbolic operators with nonsmooth coefficients. III, J. Amer. Math. Soc. 15 (2002), no. 2, 419-442 (electronic).

[16] — Null form estimates for second order hyperbolic operators with rough coefficients, in Harmonic analysis at Mount Holyoke (South Hadley, MA, 2001), Vol. 320 of Contemp. Math., 383-409, Amer. Math. Soc., Providence, RI (2003).

Department of Mathematics, University of Rochester, Rochester, NY 14627

E-mail address: dangeba@math.rochester.edu 